\title{
Anatomia endoscópica da artéria etmoidal anterior: estudo de dissecção em cadáveres
}

\section{Endoscopic anatomy of the anterior ethmoidal artery: a cadaveric dissection study}

\author{
Bernardo Cunba Araujo Filbo', Raimar Weber', \\ Carlos Diógenes Pinbeiro Neto ${ }^{3}$, Marcus Miranda \\ Lessa ${ }^{4}$, Richard Louis Voegels ${ }^{5}$, Ossamu Butugan ${ }^{6}$
}

Palavras-chave: anatomia, artéria etmoidal anterior, endoscópico, endoscópio.

Keywords: anatomy, ethmoidal anterior artery, endoscopic, endoscope.

\section{Resumo / Summary}

I

ntrodução: a artéria etmoidal anterior (AEA) é um importante ponto de reparo anatômico para localização do seio frontal e da base de crânio. Entretanto, apesar de diversos estudos endoscópicos em cadáveres, ainda não temos um estudo anatômico sobre AEA na população ocidental. Objetivos: Determinar pontos de reparo para localização da artéria, estudar sua relação com a base do crânio e grau de deiscência, assim como variações intra e interindividuais. Casuística e Métodos: Foram realizadas dissecções das fossas nasais em 25 cadáveres. Resultados: O comprimento médio do trajeto intranasal da artéria etmoidal anterior foi de $5,82 \mathrm{~mm}$. O canal etmoidal anterior apresentou algum grau de deiscência em $66,7 \%$. A distância média do ponto médio da artéria à espinha nasal anterior foi de $61,72 \mathrm{~mm}$ $(\mathrm{dp}=4,18 \mathrm{~mm})$; ̀̀ axila nasal foi de $64,04 \mathrm{~mm}(\mathrm{dp}=4,69 \mathrm{~mm})$; e à axila anterior do corneto médio foi de 21,14mm (dp = $3,25 \mathrm{~mm}$ ). Para todas as medidas, não houve diferença estatisticamente significativa quando comparados os lados direito e esquerdo ( $p>0,05)$. Conclusões: Concluímos que a axila da concha média é o ponto de reparo mais confiável para a localização da AEA.

\begin{abstract}
Introduction: The anterior ethmoidal artery (AEA) is an important point of anatomical reference in order to locate the frontal sinus and the skull base. Notwithstanding, despite numerous endoscopic studies in cadavers, we still lack an anatomical study on the AEA in the western population. Aim: to determine reference points used to locate the artery, study its relationship with the skull base and its degree of dehiscence, as well as to study intra and inter individual variations. Materials and Methods: we dissected the nasal fossae belonging to 25 cadavers. Results: the average intranasal length of the anterior ethmoidal artery was 5.2 $\mathrm{mm}$. The anterior ethmoidal canal presented some degree of dehiscence in $66.7 \%$. The average distance between the artery middle point to the anterior nasal spine was of 61.72 $\mathrm{mm}$ ( $\mathrm{sd}=4.18 \mathrm{~mm}$ ); to the lateral nasal wall (nasal axilla) was of $64.04 \mathrm{~mm}(\mathrm{sd}=4.69 \mathrm{~mm})$; and from the anterior axilla to the middle turbinate was of $21.14 \mathrm{~mm}(\mathrm{sd}=3.25 \mathrm{~mm}$ ). For all the measures there was no statistically significant measures when both sides were compared ( $p>0.05)$. Conclusions: We concluded that the middle conchae axilla is the most reliable point of reference to locate the AEA.
\end{abstract}

\footnotetext{
${ }^{1}$ Otorrinolaringologista, residência no HCFMUSP, Especialista em ORL pela SBORL, pós-graduando de doutorado da Divisão de Clinica Otorrinolaringológica do HCFMUSP. ${ }^{2}$ Médico Residente.

${ }^{3}$ Graduado em Medicina pela Faculdade de Medicina da Universidade Federal do Ceará. Residente em Otorrinolaringologia no Hospital das Clínicas da Faculdade de Medicina da USP.

${ }^{4}$ Doutor em Ciências pela Disciplina de Otorrinolaringologia da FMUSP, Fellow em Cirurgia Endoscópica Nasossinusal pela Universidade de Graz/Áustria, Prof. da Disciplina de Otorrinolaringologia da Faculdade de Medicina da UFBA.

${ }^{5}$ Professor Associado da FMUSP.

${ }^{6}$ Professor Associado da FMUSP

Trabalho realizado na Divisão de Clínica Otorrinolaringológica do Hospital das Clínicas da Faculdade de Medicina da Universidade de São Paulo. Endereço para correspondência: Bernardo Cunha Araújo Filho - Rua Oscar Freire 1799 ap. 1101 Pinheiros São Paulo SP 05409-011. E-Mail: bcaf@terra.com.br

Este artigo foi submetido no SGP (Sistema de Gestão de Publicações) da RBORL em 14 de março de 2005.

Artigo aceito em 30 de março de 2006.
} 


\section{INTRODUÇÃO}

A falta de domínio sobre a técnica cirúrgica endonasal e principalmente sobre sua anatomia foram os fatores preponderantes no elevado índice de complicações observadas nessa modalidade cirúrgica durante a década de oitenta ${ }^{1}$. Entretanto, atualmente, com a maior experiência e o conhecimento aprofundado da anatomia endoscópica nasossinusal, adquirido através da dissecção em cadáveres, as taxas de complicações graves diminuíram consideravelmente ${ }^{2}$. Desta forma, a identificação das estruturas anatômicas da cavidade nasossinusal e o reconhecimento de seus limites são essenciais para a segurança e eficácia da cirurgia endoscópica nesta região, independentemente da técnica utilizada ${ }^{3}$.

A artéria etmoidal anterior (AEA) é um importante ponto de reparo anatômico utilizado para localização do seio frontal e da base anterior de crânio ${ }^{2}$. Sua lesão inadvertida durante um procedimento endonasal pode ocasionar sérias complicações, como sangramento profuso, rinoliquorréia, retração da artéria para região intra-orbitária e conseqüentemente, hematoma orbitário e até infecções cerebrais ${ }^{4,5}$. Em raros casos (1\%) é necessário tratamento cirúrgico para o controle de epistaxe posterior, através de ligadura ou cauterização ${ }^{6}$, e em casos selecionados, com sangramentos posteriores, a artéria etmoidal anterior deve ser ligada ${ }^{7}$. Rockey et al. ${ }^{7}$ em seu estudo sobre falhas em ligaduras de artéria esfenopalatina consideram a possibilidade de sempre ligar a AEA durante o mesmo tempo cirúrgico. Woolford et al. ${ }^{3}$ propõe um procedimento endoscópico para esta ligadura, o que torna sua localização anatômica com endoscópio fundamental.

Desta forma, observamos que a localização da artéria etmoidal é muito importante para o reconhecimento de estruturas de difícil acesso (seio frontal) e demarcação do limite superior durante uma cirurgia endonasal (base do crânio). Além disso, seu achado permite, em alguns casos, reconhecer e tratar um foco de epistaxe severa.

Diferenças étnicas e entre intersexuais têm sido observada em diversos estudos e são responsáveis por grandes variações anatômicas da artéria etmoidal anterior ${ }^{2}$. Entretanto, apesar de diversos estudos endoscópicos realizados em cadáveres, ainda não temos um estudo anatômico sobre a AEA na população ocidental, no que se refere à sua relação com a base do crânio, normatizando sua localização durante cirurgias endoscópicas nasossinusais (etmoidectomia, cirurgias do seio frontal, ligadura endoscópica da AEA).

O presente estudo tem o propósito de estudar a anatomia endoscópica da artéria etmoidal anterior, através de dissecções de fossas nasais de cadáveres, identificando os pontos de referência para sua localização e as suas variações intra e interindividuais.

\section{CASUÍSTICA E MÉTODOS}

Após aprovação da Comissão de Ética para Análise dos Projetos de Pesquisa - CAPPEsq da Diretoria Clínica do Hospital das Clínicas da Faculdade de Medicina da Universidade de São Paulo (protocolo 113/04), foram realizadas dissecções em 25 cadáveres (50 fossas nasais) consecutivos no Serviço de Verificação de Óbito da Capital da Universidade de São Paulo (SVOC-USP). Dados referentes a sexo, idade, altura, peso e raça dos cadáveres foram coletados. Não foi levada em consideração a causa mortis, tendo sido incluídos indivíduos com idade superior a 18 anos. Porém, alguns critérios de exclusão foram utilizados:

- História de trauma nasal;

- Antecedentes de cirurgia nasossinusal;

- Presença de doenças que alteravam a anatomia e dificultavam a observação das estruturas nos seios esfenoidais (ex: sinusites, poliposes etc.).

\section{Métodos}

\section{Sistema de vídeo-documentação}

Todos os procedimentos foram documentados por um sistema composto por uma fonte de luz halógena (Konlux-HL2250); uma micro-câmara (Toshiva IK-CU 43a); um monitor (SEMP 10 polegadas) e uma filmadora digital (SONY Mini-DV DCR-TRV 50). Em todas as dissecções este sistema era transportado ao SVOC-USP e era responsável pela gravação de todos os procedimentos.

Para as dissecções foram utilizados materiais de cirurgia endonasal (Cottle, pinças de preensão anguladas e retas, pinças cortantes, um seeker e uma cureta para frontal), um endoscópio de 4mm 0o Storz Hopkinsâ, um endoscópio de 4mm 45o Storz Hopkinsâ e uma régua de $10 \mathrm{~mm}$ de largura e $100 \mathrm{~mm}$ de comprimento e um paquímetro para as medições (Figura 1).

\section{Técnica de dissecção}

A dissecção, sempre realizada pelo mesmo cirurgião, consistia para cada fossa nasal de uncinectomia e etmoidectomia anterior até a base anterior do crânio onde estava localizada a artéria etmoidal anterior. Em seguida eram realizados: a retirada da lâmina papirácea na região anterior adjacente à artéria etmoidal anterior e descolamento entre a lâmina papirácea e periórbita para confirmação da identificação da AEA na região onde a mesma penetrava na lâmina papirácea, através do forame etmoidal anterior (Figura 2).

Para cada lado, a trajetória intranasal da artéria foi analisada quanto à presença de deiscências do canal ósseo (totais ou parciais), após a retirada da mucosa nasossinusal sobre a artéria. Para isto foi utilizado um palpador (seeker). Com o auxílio da régua, foram medidas as distâncias do ponto médio da porção intranasal da artéria em relação à 
base do crânio, à axila anterior da concha média (extremidade anterior da inserção do corneto médio na parede lateral do nariz- AXCM), à extremidade súpero-medial da narina (região de encontro das cruras medial e lateral da cartilagem lateral inferior- AXN) e à espinha nasal anterior (ENA) (Figura 3). Além disso, sua distância à base do crânio, classificada em 3 grupos $(<2,5 \mathrm{~mm}$; $>2,5$ e $<5 \mathrm{~mm}$; $>5 \mathrm{~mm}$ ), e seu comprimento durante seu trajeto no etmóide, foram aferidos.

\section{Análise estatística}

Os dados coletados foram armazenados em um banco de dados e analisados utilizando-se o software

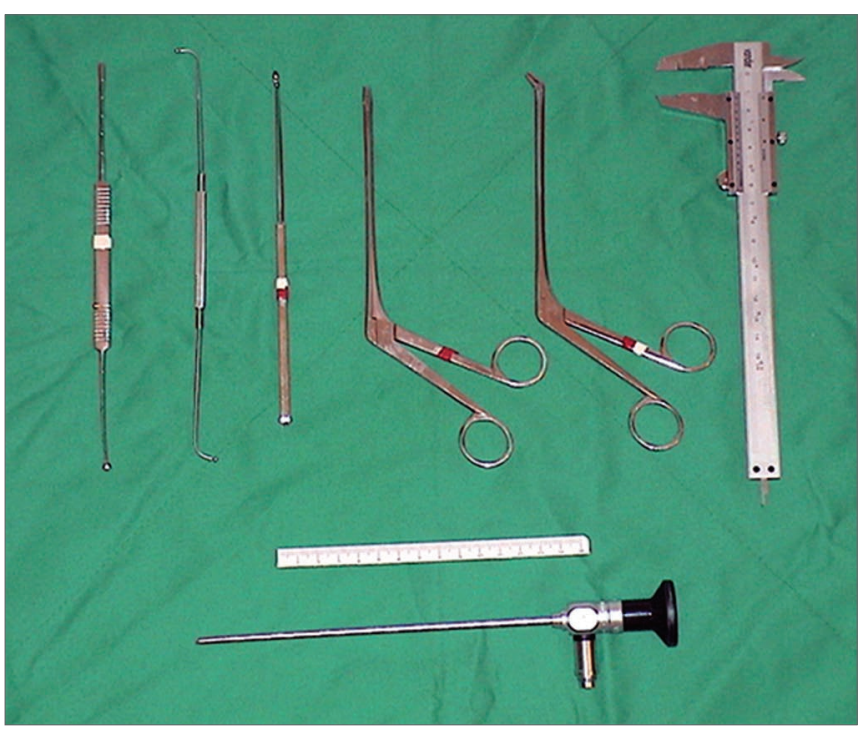

Figura 1. Material utilizado para dissecção.

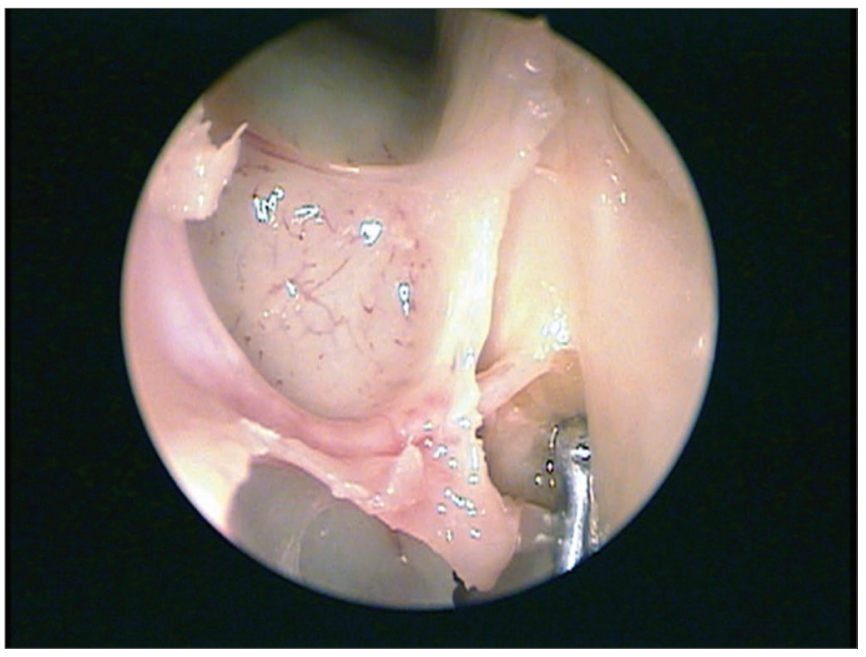

Figura 2. Artéria etmoidal anterior em fossa nasal esquerda durante a dissecção endoscópica em cadáver evidenciando deiscência completa do canal ósseo.
SPSSâ 10.0 for windows. Foi utilizado o teste de Qui-quadrado não paramétrico na comparação da prevalência de deiscência entre os lados. Para a análise das diferenças das medidas entre os sexos e entre os lados, foi utilizado o teste U de Mann-Whitney. As medidas entre os lados foram correlacionadas utilizando-se o coeficiente de correlação linear de Pearson. A concordância da presença de deiscências entre os lados foi analisada através do coeficiente de concordância Kappa. Foram considerados como estatisticamente significativos valores de "p" menores ou iguais a 0,05. Para o cálculo da amostra e intervalo de confiabilidade de 95\%, o grau de confiança foi é de $+/-5 \%$ para os valores não-paramétricos.

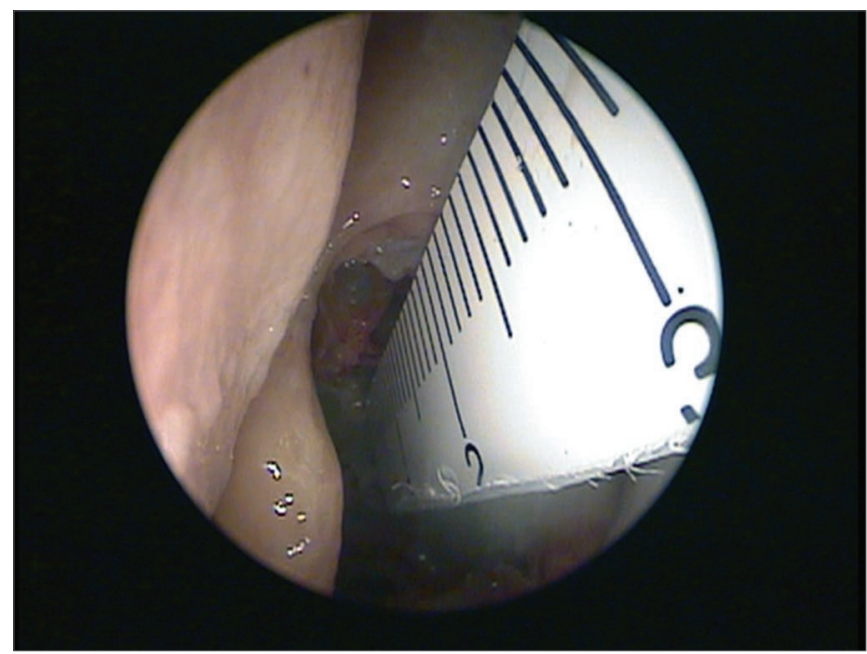

Figura 3. Medida com régua da distância da artéria etmoidal anterior à axila de concha média.

Tabela 1. Distribuição entre as raças.

\begin{tabular}{ll}
\hline RAÇA & \\
\hline Caucasóide & $52 \%$ \\
Negróide & $20 \%$ \\
Pardo & $28 \%$ \\
Total & $100 \%$ \\
\hline
\end{tabular}

Tabela 2. Presença de deiscências no canal etmoidal anterior.

\begin{tabular}{lccc}
\hline \multicolumn{4}{c}{ CANAL ETMOIDAL } \\
\hline & Frequencia & $\%$ & \% válida \\
\hline Deiscente & 12 & 24,0 & 25,0 \\
Parcialmente deiscente & 20 & 40,0 & 41,7 \\
Íntegro & 16 & 32,0 & 33,3 \\
Total & 48 & 96,0 & 100,0 \\
Não encontrada & 2 & 4,0 & \\
\hline Total & 50 & 100,0 & \\
\hline
\end{tabular}


Tabela 3. Medidas da artéria etmoidal anterior aos pontos de reparo de acordo com o sexo.

\begin{tabular}{lcccc}
\hline PONTO DE REPARO & $\mathrm{N}$ & $\begin{array}{c}\text { MÉDIA } \\
\mathrm{mm}\end{array}$ & $\begin{array}{c}\text { DESVIO } \\
\text { PADRÃO }\end{array}$ & $\begin{array}{c}\mathrm{P} \text { (Teste U de } \\
\text { Mann- Whitney) }\end{array}$ \\
\hline $\begin{array}{l}\text { Comprimento da arté- } \\
\text { ria etmoidal anterior }\end{array}$ & 48 & 5,82 & 1,41 & \\
$\begin{array}{l}\text { Masculino } \\
\text { Feminino }\end{array}$ & 19 & 5,89 & 1,62 & 0,885 \\
\hline $\begin{array}{l}\text { Distância da espinha } \\
\text { nasal anterior }\end{array}$ & 48 & 5,78 & 1,28 & \\
Masculino & 19 & 65,10 & 1,66 & 0,000 \\
Feminino & 29 & 59,51 & 3,85 & \\
\hline $\begin{array}{l}\text { Distância da axila } \\
\text { nasal anterior }\end{array}$ & 48 & 64,04 & 4,69 & \\
$\begin{array}{l}\text { Masculino } \\
\text { Feminino }\end{array}$ & 19 & 67,31 & 2,45 & 0,000 \\
\hline $\begin{array}{l}\text { Distância da axila } \\
\text { anterior do corneto }\end{array}$ & 48 & 61,89 & 4,60 & \\
$\begin{array}{l}\text { médio } \\
\text { Masculino }\end{array}$ & 19 & 21,31 & 2,84 & 0,640 \\
\begin{tabular}{l} 
Feminino \\
\hline
\end{tabular} & 29 & 21,03 & 3,54 & \\
\hline
\end{tabular}

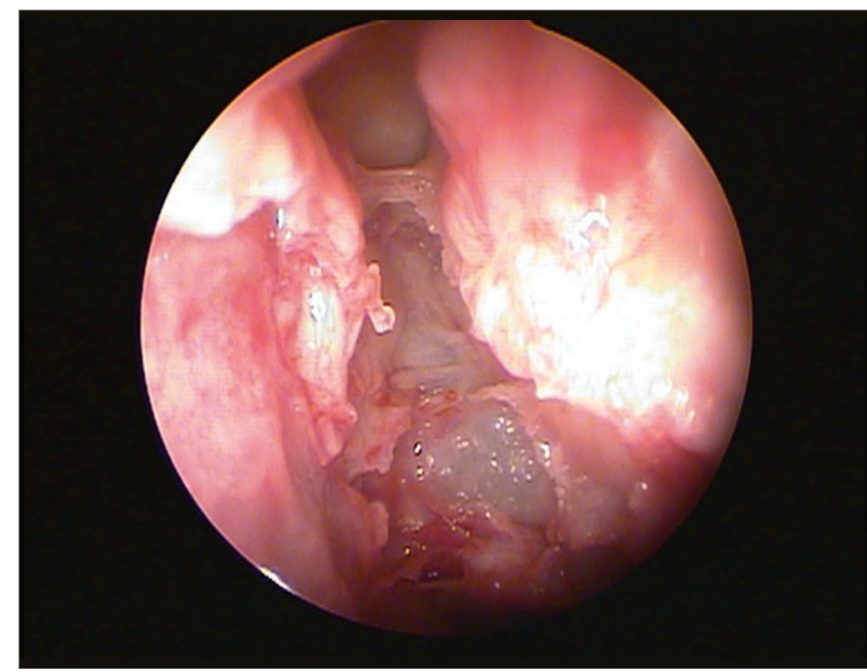

Figura 4. Presença do canal etmoidal anterior em fossa nasal cruzando o labirinto etmoidal apresentando distância superior a $5 \mathrm{~mm}$ da base do crânio (endoscópio de 45 graus).

\section{RESULTADOS}

Entre os cadáveres estudados, 10 (40\%) eram do sexo masculino e 15 (60\%) do sexo feminino. A idade variou de 39 a 83 anos (média: 60,64 $\pm 12,63$ anos). Os dados referentes à raça dos 25 cadáveres envolvidos no estudo são apresentados na Tabela 1. Entretanto, não observamos diferenças estatísticas relacionadas às medidas entre os diversos tipos raciais estudados $(p>0,05)$.

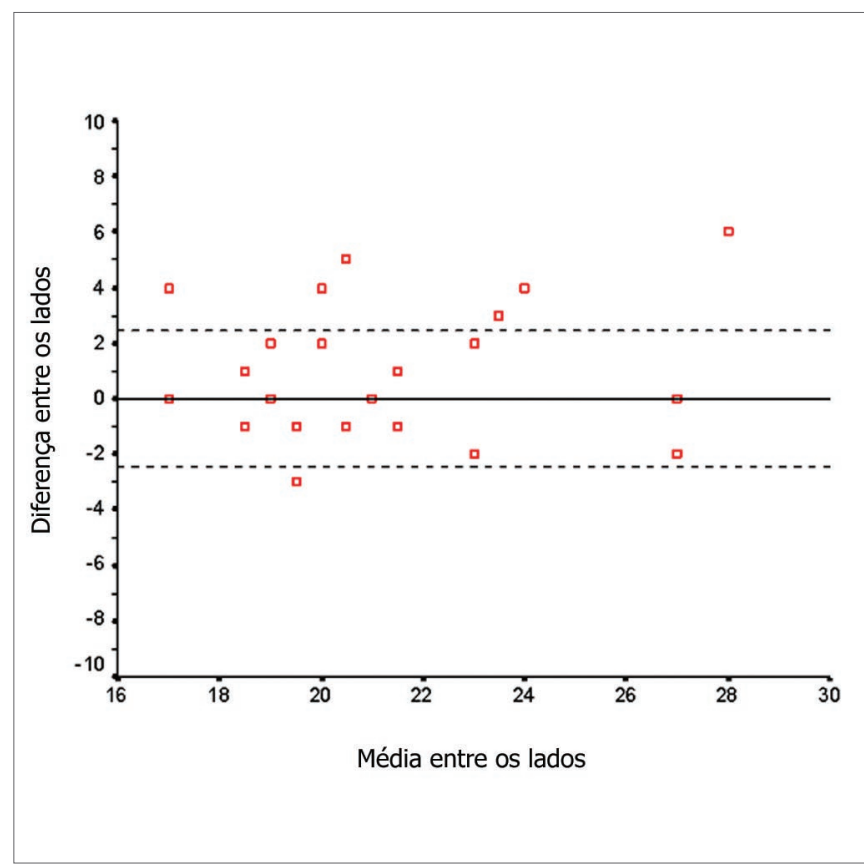

Gráfico 1. Concordância da distância da artéria etmoidal anterior à inserção anterior da concha média entre os lados direito e esquerdo.

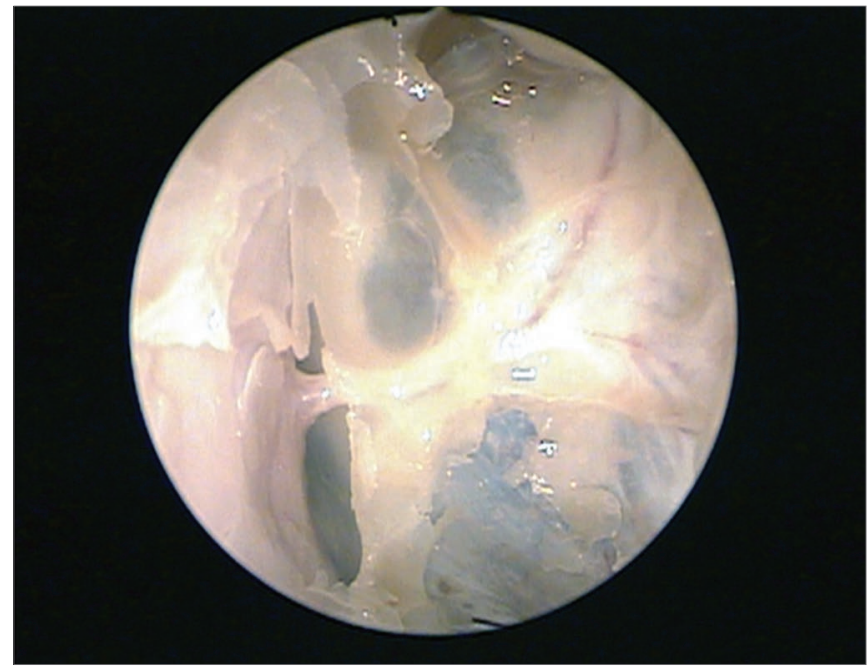

Figura 5. Artéria etmoidal anterior cruzando o labirinto etmoidal junto ao teto do etmóide anterior, completamente dentro do canal ósseo.

Estudamos 50 fossas nasais, entretanto em duas fossas não conseguimos localizar a artéria etmoidal anterior. O canal etmoidal anterior apresentou deiscência parcial em $41,7 \%$ das fossas nasais, e deiscência total em $25 \%$ (Tabela 2). O canal estava íntegro em 33,3\% dos casos. Não houve diferença estatística com relação às deiscências entre os dois sexos $\left(X^{2} \mathrm{p}=0,45\right)$. A concordância entre os lados direito e esquerdo em relação à presença de deiscências do canal etmoidal foi fraca, com coeficiente 
Kappa de 0,337; os lados foram concordantes em apenas $52 \%$ dos casos.

As distâncias da artéria etmoidal anterior em relação às referências anatômicas medidas neste estudo são apresentadas na Tabela 3. O comprimento médio do trajeto intranasal da artéria etmoidal anterior foi de $5,82 \mathrm{~mm}$ (desvio padrão $=1,41 \mathrm{~mm}$ ). A distância média do ponto médio da artéria à espinha nasal anterior foi de $61,72 \mathrm{~mm}$ $(\mathrm{dp}=4,18 \mathrm{~mm})$; ̀̀ $\mathrm{AXN}$ foi de $64,04 \mathrm{~mm}(\mathrm{dp}=4,69 \mathrm{~mm}) ; \mathrm{e}$ à AXCM foi de 21,14mm (dp = 3,25mm).

Para todas as medidas, não houve diferença estatisticamente significativa quando comparados os lados direito e esquerdo $(p>0,05)$. Fato observado na concordância entre os lados da distância da artéria etmoidal à AXCM (Gráfico 1).

Houve diferença estatística entre os sexos com relação à distância entre a artéria e a ENA e AXN ( $p<0,0001)$. O sexo masculino apresentou distância média entre a artéria e a ENA e entre a artéria e a AXN superiores às medidas encontradas no sexo feminino (Tabela 3). Entretanto, observamos que a distância entre a artéria e a AXCM não apresentou diferença estatística entre os sexos $(p=0,640)$. As médias estão apresentadas na Tabela 3. O comprimento do trajeto intranasal da artéria etmoidal anterior também não foi diferente entre os sexos $(\mathrm{p}=0,885)$.

Com relação à distância da artéria à base do crânio, 83,3\% das artérias estavam aderidas ao teto do etmóide (Figura 4 ), $4,1 \%$ entre 2,5 e $5 \mathrm{~mm}$ e $12,5 \%$ estavam localizadas a uma distância maior que $5 \mathrm{~mm}$ da base do crânio (Figura 5). Não houve diferença entre os lados $(p=0,383)$.

\section{DISCUSSÃO}

A artéria etmoidal anterior em seu trajeto intranasal encontra-se em um canal ósseo denominado canal etmoidal anterior, que parte da órbita através do forame etmoidal anterior. Esta artéria é responsável pela irrigação de células etmoidais anteriores, seio frontal, emite vasos meníngeos em seu trajeto na fossa olfatória e desce para a fossa nasal, onde irriga o terço anterior do septo e da parede lateral do nariz adjacente 4 . Esta artéria percorre o teto etmoidal em direção diagonal, em sentido pósteroanterior (Figura 2) e o local onde penetra no crânio (união da placa cribiforme com a lamela lateral da fossa olfatória) é a região mais frágil e susceptível às lesões, causando fístula liquórica ${ }^{8,9}$.

Vários estudos têm elaborado técnicas para o reconhecimento da AEA. Kirchner et al. ${ }^{8}$ estudou a anatomia da AEA, porém útil em uma abordagem externa, não endoscópica. Stammberger et al. ${ }^{10}$ sugeriu que a AEA estaria em um ponto $1 \mathrm{a} 2 \mathrm{~mm}$ posterior ao ponto mais superior da face anterior da bolha etmoidal, enquanto Lund et al. ${ }^{11}$ propõe que a parede posterior do recesso frontal é o ponto de reparo para localização da artéria. Porém, estes estudos apontam possíveis diferenças étnicas e nem sempre são uma regra.

Em nosso estudo, houve algum grau de deiscência da artéria em quase dois terços (66\%) das fossas nasais dissecadas. Este achado reforça a importância do conhecimento da localização da artéria etmoidal anterior durante as cirurgias endonasais, uma vez que a falha na integridade do canal ósseo torna a artéria mais suscetivel às lesões inadvertidas. A presença de deiscência da artéria em uma fossa nasal durante uma cirurgia não pode ser utilizada como fator preditivo para a deiscência na fossa contralateral, pois que em apenas $52 \%$ dos cadáveres havia concordância. Moon et al. ${ }^{3}$ e Stammberger et al. ${ }^{8}$ apresentaram em seus estudos graus menores de deiscências (11,4\% e $40 \%$, respectivamente). O fato de termos considerado deiscências parciais e também as diferenças raciais pode explicar esta discrepância.

Em duas fossas nasais não foi possível localizar a AEA. Lee et al. ${ }^{2}$ consideraram haver ausência de artéria etmoidal, porém não encontrou este achado em seu estudo realizado apenas entre chineses. Isaacson et al. ${ }^{12}$ estudando crânios em crianças relataram ausência do forame etmoidal anterior em 5\% dos casos. Há relatos que a artéria etmoidal pode estar ausente ou seu percurso ocorre por dentro da parede óssea da base de crânio ${ }^{13}$, sendo impossível de localizá-la, entretanto não creditamos a ausência da artéria a este último fato, pois confirmávamos sua presença em seu trajeto intra-orbitário (fora de seu trajeto ósseo).

O presente estudo mostrou que a artéria etmoidal anterior encontra-se em média a $21,14 \mathrm{~mm}$ da axila anterior do corneto médio, a $61,72 \mathrm{~mm}$ da espinha nasal anterior, e a $64,04 \mathrm{~mm}$ da extremidade súpero-medial da narina. Tais medidas foram muito semelhantes às encontradas por Lee et al. ${ }^{2}$. Os mesmos autores descreveram uma relação linear entre a extremidade súpero-medial da narina, a axila anterior do corneto médio e a artéria etmoidal anterior (Figura 3), entretanto não concordamos com este achado. Observamos que esta reta encontrava o crânio 3 a $4 \mathrm{~mm}$ posterior a artéria etmoidal anterior (AEA). Em seu estudo, Lee et $\mathrm{al}^{2}{ }^{2}$ retirava a região equivalente à mucosa da axila do corneto médio, o que, possivelmente anteriorizava sua medida. Moom et al. e Lee et al. em estudos semelhantes utilizaram endoscópios de 0 grau nas dissecções, o que ao nosso entender dificultou bastante a localização da AEA, muitas vezes localizada em nossa casuística apenas com endoscópio de 45 graus. A relação linear é válida, porém devemos considerar a superestimativa posterior desta regra.

As medidas encontradas no presente estudo foram significativamente diferentes entre os sexos, sendo maiores no masculino, discordando dos dados observados em outros estudos de dissecção ${ }^{2,4}$. Apenas a distância da AEA à AXCM não apresentou diferenças entre os sexos e entre os lados direito e esquerdo, e seu desvio padrão foi o menor observado entre as medidas de distância en- 
tre os indivíduos. Podemos, desta forma, considerar esta medida bastante útil e confiável na prática clínica para localização da AEA.

Em nosso estudo, $12,5 \%$ das AEA estavam a mais de $5 \mathrm{~mm}$ distante do teto do etmóide, fato também observado por Moom et al. (14,3\%). Isto permite inferir que nosso método de avaliação foi satisfatório para este item, mesmo sem utilizarmos cortes sagitais (de estudo mais apurado) de cabeças, como realizado por Moom et al. Este fato torna a cirurgia do etmóide mais sujeita às lesões da AEA. Uma exploração endoscópica criteriosa, com atenção do teto do etmóide é necessária para evitar possíveis traumas e seqüelas irreparáveis, já comentadas anteriormente.

Ohnishi et al. ${ }^{4}$ consideram a possibilidade de cantotomia lateral e Lynch para tratar possíveis lesões iatrogênicas da AEA. Entretanto, baseados em nossa metodologia podemos considerar que a ligadura deste vaso pode ser realizada por via endoscópica, como também preconizado por Woolford et al. ${ }^{14}$, ou por uma micro incisão em pele e auxílio do endoscópio, proposta de Douglas et al. ${ }^{15}$.

\section{CONCLUSÕES}

- A axila do corneto médio é o ponto de reparo mais confiável para a localização da AEA, estando em média a $21 \mathrm{~mm}$ desta, não observando diferença entre o sexo e entre os lados.

- Em 12,5\% dos casos de nossa amostra o risco de lesão inadvertida da artéria etmoidal anterior durante uma cirurgia funcional dos seios paranasais é maior, por esta se encontrar a mais de $5 \mathrm{~mm}$ do teto etmoidal.

- Em 66,7\% dos casos, o canal etmoidal anterior apresenta algum grau de deiscência. Desta forma, a cirurgia endoscópica nasossinusal em etmóide deve ser criteriosa, estabelecendo limites, a fim de evitar complicações mais graves.

\section{REFERÊNCIAS BIBLIOGRÁFICAS}

1. Stankiewz JA. Complications of endoscopic intranasal ethmoidectomy. Laryngoscope 1987;97:1270-3.

2. Lee WC, Ku PKM, Hasselt CA. New guidelines for endoscopic localization of the anterior ethmoidal artery: a cadaveric study. Laryngoscope 2000;110:1173-8.

3. Moon Hyoung-Jin, Hyung-Ung Kim, Jeung-Gweon Lee, In Hyuk Chung. Surgical anatomy of the anterior ethmoidal canal in ethmoid roof. Laryngoscope 2001;111:900-4.

4. Ohnishi T, Yanagisawa E. Endoscopic anatomy of the anterior ethmoidal artery. Ear Nose and Throat Journal 1994:634-6.

5. Voegels RL. Cirurgia endoscópica dos seios paranasais. Arquivos de otorrinolaringologia 1997;1(1).

6. Brouzas D, Charakidas A, Androulakis M, Moschos M. Traumatic optic neuropathy after posterior ethmoidal artery ligation for epistaxis. Otolaryngology - Head and Neck Surgery 2002;126 (4):323-25.

7. Rockey JG, Anand R. A critical audit of the surgical management of intractable epistaxis using sphenopalatine artery ligation/diathermy. Rhinology 2002;40:147-149.

8. Kirchner JA, Yanagisawa E, Crelin ES. Surgical anatomy of the ethmoidal arteries. Arch Otolaryngol 1961;74:382-6.

9. Basak S, Karamam CZ, Mutlu C, Odabasi O. Evaluation of some important anatomical variations and dangerous areas of the paranasal sinuses by CT for safer endonasal surgery. Rhinology 1998;36:1627.

10. Stammberger H. Functional Endoscopic Sinus Surgery: The Messerklinger Technique. St. Louis, MO: Mosby-Year Book; 1991.p.706.

11. Lund VJ. Anatomy of the nose and paranasal sinuses. In: Gleeson M, ed. Scott Brown's Otolaryngology, 6th ed. London: Butterworths, 1996.

12. Isaacson G, Monge JM. Arterial ligation for pediatric epistaxis: developmental anatomy. American Journal of Rhinology 2003;17(2):75-81.

13. Becker SP. Applied anatomy of the paranasal sinuses with emphasis on endoscopic surgery. Ann Otol Rhinol Laryngol Suppl 1994;162:332.

14. Woolford TJ, Jones NS. Endoscopic ligation of anterior ethmoidal artery in treatment of epistaxis. The Journal of Laryngology \& Otology 2000;114:858-60.

15. Douglas SA, Gupta D. Endoscopic assisted esternal approach anterior ethmoidal artery ligation for management of epistaxis. The Journal of Laryngology \& Otology 2003;117:132-3. 